\title{
Instrumentos de avaliação de qualidade de vida de pessoas com estomias intestinais: revisão integrativa
}

\author{
Instruments for the evaluation of quality of life of people with intestinal \\ stomies: integration review
}

\author{
Prisciane Cardoso Silva' • Marina Soares Mota \\ Aline Neutzling ${ }^{3}$ Caroline Betanzos Amorim ${ }^{4}$ \\ Bianca dos Santos Blan ${ }^{5}$ Stella Minasi de Oliveira ${ }^{6}$
}

\begin{abstract}
RESUMO
Objetivo: Buscar na literatura instrumentos utilizados para avaliar a qualidade de vida de pessoas com estomias intestinais. Metodologia:Trata-se de uma revisão integrativa realizada no ano de 2019, em bases de dados nacionais e internacionais. Resultados: Foram encontrados 17 artigos, com sete instrumentos utilizados para avaliar a qualidade de vida de pessoas com estomias intestinais: City of Hope Quality of Life-Ostomy Questionnaire, Stoma Self-Efficacy Scale, World Health Organization Quality of Life abreviado, European Organization for Research and Treatment of Cancer Quality of Life Questionnaire, Ostomy-specific (Stoma-QoL) e Escala de Qualidade de Vida de Flanagan. Conclusão: Esta revisão permitiu identificar os instrumentos que estão sendo utilizados para avaliar a QV de pessoas com estomias intestinais.Após a análise dos instrumentos, salienta-se que o City of Hope - Quality of Life - Ostomy Questionnaire é o mais abrangente dentre os instrumentos específicos às pessoas com estomias intestinais.

Descritores: Qualidade de Vida; Estomias; Enfermagem médico-cirúrgica.
\end{abstract}

\begin{abstract}
Objective:To search the literature for instruments used to evaluate the quality of life of people with intestinal tumors. Methodology: This is an integrative review carried out in 2019, in national and international databases. Results: We found 17 articles, with seven instruments used to evaluate the quality of life of people with intestinal stomies: City of Hope Quality of Life-Ostomy Questionnaire, Stoma Self-Efficacy Scale, World Health Organization Quality of Life abbreviated, European Organization for Research and Treatment of Cancer Quality of Life Questionnaire, Ostomyspecific (Stoma-QoL) and Flanagan Quality of Life Scale. Conclusion:This review allowed to identify the instruments that are being used to evaluate the QoL of people with intestinal stomies. After analyzing the instruments, the City of Hope - Quality of Life - Ostomy Questionnaire is the most comprehensive of the instruments specific to people with intestinal stomies.
\end{abstract}

Descriptors: Quality of Life; Stomias; Medical-surgical nursing.

' Possui graduação em Enfermagem pela Universidade Federal do Rio Grande (2014). Especialista na Atenção à Saúde Cardiometabólica do Adulto pela Residência Integrada Multiprofissional Hospitalar com ênfase na atenção à saúde cardiometabólica do adulto (2017). Trabalhou como enfermeira assistencial - Unidade de Terapia Intensiva e Centro de Assistência a Queimados na Associação de Caridade Santa Casa do Rio Grande. Cursando Mestrado em Enfermagem e Saúde na Universidade Federal do Rio Grande.Atualmente trabalha como enfermeira assistencial na Unidade de Pronto Atendimento - UPA/Areal, Pelotas. Email: priscianecardososilva@gmail.com

2 Possui graduação em Enfermagem pela Universidade Federal do Rio Grande (2012), mestrado (2014) e doutorado (2017) pelo Programa de Pós-graduação em Enfermagem com ênfase no contexto socioambiental da Universidade Federal do Rio Grande (FURG). Tem experiência na área de Enfermagem em saúde mental, atendimento em estomaterapia e em urgência e emergências.Atualmente, é Professora Adjunta da FURG ministrando as disciplinas de Semiologia e Semiotécnica II e Saúde Ambiental. Tutora quilombola pelo Acompanhamento e Apoio Pedagógico ao Estudante da FURG. Membro efetivo do Programa de Pós-graduação em Enfermagem e do Conselho da Escola de Enfermagem da FURG.Vice-líder do Grupo de Estudos e Pesquisas em Enfermagem na Saúde da Criança e do Adolescente/G

${ }_{3}^{3}$ Possui graduação em Licenciastura Plena em Ciencias Biologicas pela Universidade Católica de Pelotas (1997),mestrado em Tropical International Agriculture - Agrobusiness (UTALCA/GAUG) - Georg August Universitaet Goettingen (2009), doutorado em Agricultural Sciences - Georg August Universitaet Goettingen (2014). Pós-doutorado em Enfermagem pela Universidade Federal de Pelotas (2018). Atualmente é professora visitante da Universidade Federal do Rio Grande. Tem experiência na área de Saúde Coletiva, com ênfase em epidemiologia e análises quantitativas em ciências da saúde.

${ }^{4}$ Enfermeira Graduada pela Universidade Federal do Rio Grande -FURG (2017). Integrante do Núcleo de Estudos e Pesquisas em Enfermagem e Saúde - NEPES da FURG. Mestranda do Programa de Pós-Graduação em Enfermagem da Universidade Federal do Rio Grande - PPGEnf/FURG.

${ }^{5}$ Formanda de Enfermagem dedicada e determinada a fazer a diferença e lutar em prol de um Sistema Único de Saúde de qualidade. Durante a graduação desenvolvi maior afinidade ao cuidado à pessoas com estomias, enfermagem gerítrica, cuidado

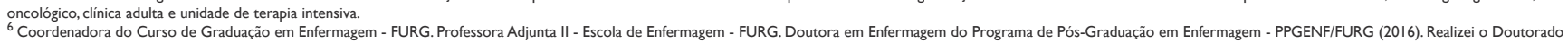
sanduiche no exterior - Bolsista PDSE - CAPES, na Universitat Di Roviri IVirgili - URV, Campus Tortosa/Tarragona - Espanha. Mestrado em Enfermagem pela FURG (2003), Especialização Multiprofissional Saúde da Familia pela FURG (2003), Graduação em

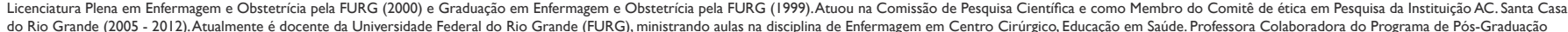
do Rio Grande (2005 - 2012). Atualmente e docente da Universidade Federal do Rio Grande (FURG), ministrando aulas na disciplina de Enfermagem em Centro Cirúrgico, Educação em Saúde. Professora Colaboradora do Programa de Pos-Graduaç̧a Educação em Saúde, atuando principalmente nos seguintes temas: cuidado à saúde da criança e do adolescente, saúde da família, centro cirúrgico, cuidado em enfermagem. 


\section{INTRODUÇÃO}

Qualidade de vida $(\mathrm{QV})$ é um termo amplo e referese à percepção do indivíduo sobre seu estado de saúde em relação aos aspectos sociais, físicos, psicológicos, econômicos e espirituais ${ }^{(1)}$. A QV ligada à saúde é o valor atribuído à vida com base nas deteriorações funcionais, percepções e condições sociais induzidas por doenças, agravos e tratamentos, além da organização política e econômica do sistema assistencial(2).

A preocupação com um conceito de $\mathrm{QV}$ se deve a um movimento dentro das ciências humanas e biológicas, que busca valorizar parâmetros que vão além de controle de sintomas, diminuição de mortalidade ou aumento de expectativa de vida. Um movimento para humanizar a saúde e a QV, com a ideia de acrescentar vida aos anos e não apenas anos à vida dos indivíduos $^{(3)}$.

A QV é definida como a percepção ampla e abrangente do indivíduo em relação ao seu contexto cultural e de valores, por meio da relação das suas metas, expectativas, padrões e preocupações ${ }^{(4)}$. A introdução desse conceito foi uma importante contribuição para as medidas de desfecho em saúde, tendo um valor intrínseco e intuitivo devido à sua abrangência e ligação com o que o próprio indivíduo sente e percebe. Independente de nação, cultura ou época, é importante que as pessoas se sintam bem psicologicamente, possuam boas condições físicas e sintam-se socialmente integradas e funcionalmente competentes ${ }^{(5)}$.

A partir do estabelecimento de um conceito, tornou-se possível a criação e a aplicação de instrumentos de avaliação da $\mathrm{QV}$, que inclui, além da prática clínica individual, a avaliação da efetividade de tratamentos e funcionamento de serviços de saúde. Os instrumentos de avaliação também são importantes guias para a formulação de políticas de saúde, permitindo o desenvolvimento de estratégias eficientes e eficazes para a promoção da qualidade de vida da população(6).

Nesse contexto, a avaliação da QV se torna extremamente benéfico às pessoas com estomias intestinais ao permitir a formulação do cuidado ampliado em saúde para além das questões físicas, mas também abordando as dimensões sócias e psicológicas. Denomina-se como pessoa com estomia aquela que, devido a um procedimento cirúrgico resultou na exteriorização de um sistema (digestório, respiratório e urinário), a qual possui um estoma, ou seja, uma abertura artificial entre os órgãos internos para o meio externo(7).

Especificamente, as estomias intestinais têm por principal finalidade o desvio da eliminação de fezes e gazes, por meio da exteriorização do cólon (colostomia) ou do íleo (ileostomia)(8). Essa condição pode levar à insatisfação com o próprio corpo, baixa autoestima, sentimentos de auto exclusão, além de depressão e dificuldades de retornar aos hábitos valorizados e prazerosos como atividades laborais e de bem-estar, rotinas diárias, reinserção social e da sexualidade (9).

Nesse sentido, o cuidado de enfermagem voltado a essas pessoas torna-se essencial e pauta-se nas mudanças concretas que a estomia acarreta em suas vidas, permitindo que elas adquiram conhecimentos acerca da doença de base que gerou a estomia, dos cuidados voltados ao estoma e pele periestomal, de como melhorar a QV por meio da promoção do autocuidado, existência de uma rede se apoio, estímulo à inserção em grupo terapias para troca de conhecimentos, segurança e lazer ${ }^{(10)}$.

Assim, a elaboração de instrumentos técnicos que avaliem características da população devem satisfazer determinadas condições e requerem procedimentos padronizados. Desse modo, se faz necessário a evidenciação e análise de suas propriedades psicométricas, uma vez que um instrumento que não atende tais princípios não pode ser reconhecido cientificamente ${ }^{(1)}$.

Portanto, um bom instrumento é aquele que permite fácil aplicação, pontuação e interpretação, além de tomar o menor tempo possível, para ambas as partes envolvidas: examinador e examinado. Ainda, o instrumento deve ser relevante e adequado à população aplicada e possuir indicativos de precisão e validade conexo com os objetivos pretendidos na avaliação(12).

Ao longo dos anos, muitos pesquisadores avaliaram o impacto de uma estomia na vida das pessoas utilizando metodologias qualitativas ${ }^{(13)}$. Durante a década de 1980, quando muitos desses estudos foram publicados, o Índice de Qualidade de Vida, criado por Padilla e Grant, foi desenvolvido e avaliado quanto à validade e confiabilidade ${ }^{(14)}$. A partir de então, diversos instrumentos específicos passaram a ser desenvolvidos para medir a qualidade de vida relacionada à saúde (QVRS) de pessoas com estomia ${ }^{(13)}$. Assim, o presente estudo teve como objetivo buscar na literatura os instrumentos utilizados para avaliar a QV de pessoas com estomias intestinais, a fim de nortear futuras avaliações.

\section{METODOLOGIA}

Trata-se de uma revisão integrativa, que reúne e sintetiza resultados de pesquisas de um determinado tema, com o intuito de contribuir para a disseminação de conhecimento. Seis etapas foram percorridas para a presente revisão: identificação do tema; estabelecimento dos critérios para a inclusão e exclusão dos artigos (seleção da amostra); leitura dos títulos e resumos; categorizações dos estudos de interesse pela técnica de fichamento; leitura exaustiva e análise dos resultados; discussão e apresentação dos estudos incluídos de acordo com o interesse proposto pelo estudo ${ }^{(15)}$.

Como critérios de inclusão, destacaram-se: artigos científicos publicados em periódicos indexados, no período de 2014-2019; originais; nos idiomas português, inglês, espanhol; artigos que utilizassem instrumentos de avaliação de qualidade de vida de pessoas com estomias intestinais $>18$ anos; artigos livres, disponíveis na íntegra.

Foram excluídos da pesquisa revisões, teses, dissertações e materiais técnico-dissertativos, além de artigos que não utilizassem instrumentos de avaliação de qualidade de vida de pessoas com estomias intestinais, ou que os utilizasse em crianças; artigos parcialmente disponíveis.

Desta forma, determinou-se como questão norteadora "Quais os instrumentos utilizados para avaliar a qualidade de vida das pessoas com estomias intestinais?". A busca dos dados ocorreu entre os meses de fevereiro e março de 2019 e a pesquisa deu-se em conformidade com as especificidades de cada base de dados em pesquisa.A combinação dos descritores do Medical SubjectHeading (MESH) "quality of life", "ostomy" e "Medical-Surgical Nursing" foi utilizada para a busca na base de dados SciVerseScopus (Scopus) e na Medical Literature Analysis and Retrieval System Online (MedLine).

Enquanto que para as buscas realizadas na Literatura LatinoAmericana e do Caribe em Ciencias da Saúde (Lilacs), Base de dados em Enfermagem (Bdenf), Scientific Electronic Library Online (SciELO) utilizaram-se, além dos descritores do Mesh, também os termos dos Descritores em Ciências da Saúde 
(DecS) em português/espanhol: "qualidade de vida/calidad de vida", "estomias/estomías" e "enfermagem médico-cirúrgica/ enfermería médico-quirúrgica". Utilizou-se em todas as bases de dados o operador booleano "AND". As estratégias de busca são apresentada no Quadro I.

Cada estudo foi avaliado de acordo com seu nível de evidência, conforme a classificação demonstrada no Quadro 2.

Quadro I - Estratégias de Busca

\begin{tabular}{|c|c|}
\hline Base de Dados & Estratégias de Busca \\
\hline Scopus & $\begin{array}{l}\text { "Quality of life” (mesh) and “Os- } \\
\text { tomy” (mesh) “Medical-Surgical } \\
\text { Nursing” (mesh). }\end{array}$ \\
\hline Medline & $\begin{array}{l}\text { "Quality of life" (mesh) and “Os- } \\
\text { tomy” (mesh) "Medical-Surgical } \\
\text { Nursing” (mesh). }\end{array}$ \\
\hline Lilacs & $\begin{array}{l}\text { "Qualidade de vida/Calidad de vida”, } \\
\text { (Decs português/espanhol) and } \\
\text { "Estomias/Estomías" (Decs portu- } \\
\text { guês/espanhol) and "Enfermagem } \\
\text { médico-cirúrgica/Enfermería méd- } \\
\text { ico-quirúrgica" (Decs português/ } \\
\text { espanhol). }\end{array}$ \\
\hline Bdenf & $\begin{array}{l}\text { "Qualidade de vida/Calidad de vida”, } \\
\text { (Decs português/espanhol) and } \\
\text { "Estomias/Estomías" (Decs portu- } \\
\text { guês/espanhol) and "Enfermagem } \\
\text { médico-cirúrgica/Enfermería méd- } \\
\text { ico-quirúrgica” (Decs português/ } \\
\text { espanhol). }\end{array}$ \\
\hline Scielo & $\begin{array}{l}\text { "Qualidade de vida/Calidad de vida”, } \\
\text { (Decs português/espanhol) and } \\
\text { "Estomias/Estomías" (Decs portu- } \\
\text { guês/espanhol) and "Enfermagem } \\
\text { médico-cirúrgica/Enfermería méd- } \\
\text { ico-quirúrgica” (Decs português/ } \\
\text { espanhol). }\end{array}$ \\
\hline
\end{tabular}

Quadro 2 - Nível de evidência

\begin{tabular}{|c|c|}
\hline I & $\begin{array}{c}\text { Revisão sistemática ou metanálise de múltiplos estudos } \\
\text { clínicos randomizados. }\end{array}$ \\
\hline II & Ensaios clínicos randomizados bem desenhados. \\
\hline III & Ensaios clínicos bem desenhados sem randomização. \\
\hline IV & Estudos de caso-controle e de coorte. \\
\hline V & Revisões sistemáticas de estudos descritivos e qualitativos. \\
\hline VI & Estudos descritivos ou de abordagem qualitativa. \\
\hline VII & Opinião de especialistas. \\
\hline
\end{tabular}

\section{RESULTADOS}

A partir da pesquisa nas bases de dados, foram encontrados 22 artigos na Lilacs, I 3 na Scielo e 594 na Scopus. Nas bases BedEnf e MedLine não foram encontrados artigos com os descritores utilizados. Após a leitura dos resumos, foram selecionados 323 artigos nas bases referidas, para serem analisados na íntegra e submetidos aos critérios de inclusão e exclusão. Durante a análise, 306 artigos foram excluídos, obtendo o resultado de 17 artigos para a discussão de conteúdo (Figura I).
Figura I. Fluxograma do processo de inclusão dos estudos.
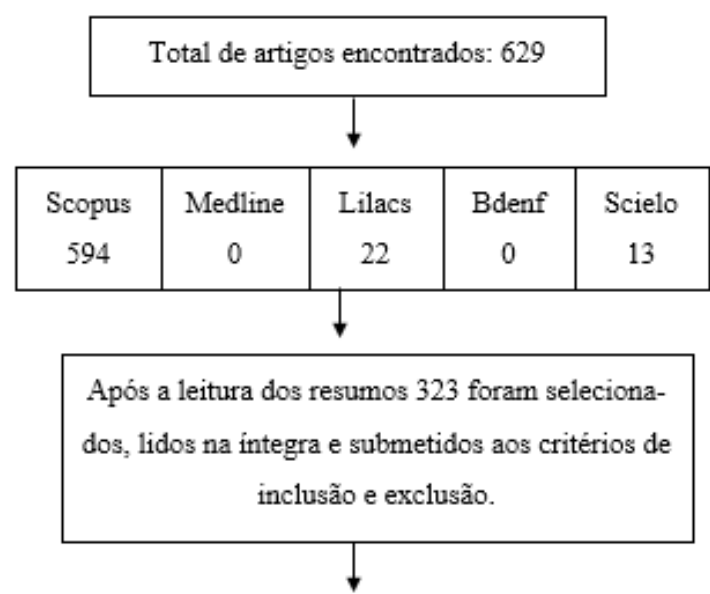

Mo6 foram excluidos.
Motivo da exclusão:
- Artigos repetidos: 43
- Revisões, teses, dissertações, artigos que não utili-
zaram instrumentos de avaliação de QV de pessoas
com estomias intestinais, ou foi utilizado em crian-
ças, artigos parcialmente disponiveis: 263

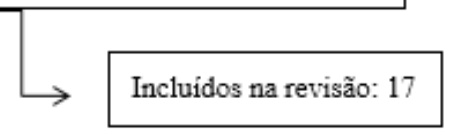

Fonte: Elaboração Própria

Dos 17 artigos, 14 (82\%) foram publicados em inglês, os três restantes foram publicados em português (I8\%). No que se refere ao país de origem do estudo, o Brasil tem 10 publicações (58,8\%), seguido pela Holanda com duas (I I,7\%) e Alemanha, China, Estados Unidos da América, Sérvia e Turquia com uma publicação cada $(5,9 \%$ cada).

Em relação à condição que levou à estomização dos participantes dos estudos em questão, as neoplasias são as causas mais frequentes, sendo citadas em 14 desses (82\%). No entanto, também são citadas outras causas (18\%) como a colite ulcerosa e Doença de Crohn, uma vez cada em estudos diferentes e fístula, diverticulite e doença inflamatória intestinal aparecem uma vez cada, no mesmo estudo, juntamente com a neoplasia. Trauma e perfuração intestinal aparecem também uma vez cada em diferentes estudos.

O quadro 3 descreve os 17 estudos em relação ao país e ano de publicação, título, instrumento de avaliação de qualidade de vida utilizado, causa da estomia intestinal dos participantes e a conclusão dos mesmos. E o quadro 4 apresenta os níveis de evidência dos estudos.

Foram encontrados sete instrumentos utilizados para avaliar a QV de pessoas com estomias intestinais, sendo eles: City of Hope Quality of Life-Ostomy Questionnaire (COHQOL-OQ), utilizado em seis estudos (35,2\%), sendo um associado à Stoma Self-Efficacy Scale (SSES), seguido pelo World Health Organization Quality of Life abreviado (WHOQOL-BREF), utilizado em cinco estudos (29,4\%). O instrumento European Organization for Research and Treatment of Cancer Quality of Life Questionnaire (EORTC QLQ-C30) foi utilizado em três estudos (17,7\%) e o 
Ostomy-specific (Stoma-QoL) foi utilizado em dois (II,8\%). A Escala de Qualidade deVida de Flanagan foi utilizada em apenas um estudo $(5,9 \%)$ dessa revisão.

O quadro 4 descreve a avaliação dos estudos em relação ao nível de evidência. Foram encontrados 16 artigos com nível de evidência VI e apenas um estudo com nível de evidência III.

Quadro 3. Características dos estudos sobre qualidade de vida de pessoas com estomias intestinais, Rio Grande, RS, Brasil. 2019.

\begin{tabular}{|c|c|c|c|c|c|}
\hline $\mathbf{N}^{\circ}$ & País/Ano & Título & $\begin{array}{l}\text { Instru- } \\
\text { mento }\end{array}$ & $\begin{array}{l}\text { Causa da } \\
\text { estomia }\end{array}$ & Conclusão \\
\hline 1 & $\begin{array}{l}2014 \\
\text { Brasil }\end{array}$ & $\begin{array}{l}\text { Quality of life and } \\
\text { self-esteem of } \\
\text { patients with intesti- } \\
\text { nal stoma }\end{array}$ & $\begin{array}{l}\text { Escala de } \\
\text { Qualidade } \\
\text { deVida de } \\
\text { Flanagan }\end{array}$ & $\begin{array}{l}\text { Neoplasia é } \\
\text { a causa mais } \\
\text { frequente }\end{array}$ & $\begin{array}{l}\text { Os pacientes deste es- } \\
\text { tudo mostraram baixa } \\
\text { autoestima e QV. }\end{array}$ \\
\hline 2 & $\begin{array}{l}2014 \\
\text { Brasil }\end{array}$ & $\begin{array}{l}\text { The influence of } \\
\text { time on the quality } \\
\text { of life of patients } \\
\text { with intestinal } \\
\text { stoma }\end{array}$ & $\begin{array}{l}\text { WHO } \\
\text { QOL- } \\
\text { BREF }\end{array}$ & Não referido & $\begin{array}{l}\text { Pacientes com ostomia } \\
\text { há mais de dois anos } \\
\text { têm melhor QV. }\end{array}$ \\
\hline 3 & $\begin{array}{l}2015 \\
\text { Holanda }\end{array}$ & $\begin{array}{l}\text { A mixed-method } \\
\text { study on the } \\
\text { generic and osto- } \\
\text { my-specific quality } \\
\text { of life of cancer and } \\
\text { non-cancer ostomy } \\
\text { patients }\end{array}$ & $\begin{array}{c}\text { Sto- } \\
\text { ma-QoL }\end{array}$ & $\begin{array}{l}\text { Neoplasia, } \\
\text { colite ulcero- } \\
\text { sa, Doença de } \\
\text { Crohn }\end{array}$ & $\begin{array}{l}\text { Pacientes oncológicos } \\
\text { relataram melhor QV } \\
\text { que pacientes com } \\
\text { estomia não oncológica }\end{array}$ \\
\hline 4 & $\begin{array}{l}2016 \\
\text { Brasil }\end{array}$ & $\begin{array}{l}\text { Quality of life } \\
\text { in stomized } \\
\text { oncological patients: } \\
\text { an approach of } \\
\text { integrality from } \\
\text { Brazilian Unified } \\
\text { Health System }\end{array}$ & $\begin{array}{l}\text { WHO } \\
\text { QOL- } \\
\text { BREF }\end{array}$ & $\begin{array}{l}\text { Neoplasia } \\
\text { colorretal }\end{array}$ & $\begin{array}{c}\text { A atenção integral à } \\
\text { saúde promove melho- } \\
\text { rias na QV. }\end{array}$ \\
\hline 5 & $\begin{array}{l}2016 \\
\text { Brasil }\end{array}$ & $\begin{array}{l}\text { Sociodemographic } \\
\text { and clinical features } \\
\text { and quality of life in } \\
\text { stomized patients }\end{array}$ & $\begin{array}{c}\mathrm{COH} \\
\mathrm{QOL-OQ}\end{array}$ & $\begin{array}{l}\text { Neoplasia é } \\
\text { a causa mais } \\
\text { frequente }\end{array}$ & $\begin{array}{l}\text { Os participantes do } \\
\text { estudo possuíam QV } \\
\text { satisfatória. }\end{array}$ \\
\hline 6 & $\begin{array}{c}2016 \\
\text { Alemanha }\end{array}$ & $\begin{array}{l}\text { Quality of life and } \\
\text { need for care in } \\
\text { patients with an } \\
\text { ostomy: a survey } \\
\text { of } 2647 \text { patients } \\
\text { of the Berlin OSto- } \\
\text { my-Study (BOSS) }\end{array}$ & $\begin{array}{l}\text { EORTC } \\
\text { QLQ-C30 }\end{array}$ & Neoplasia & $\begin{array}{c}\text { O cuidado contínuo } \\
\text { e as informações } \\
\text { oferecidas ao paciente } \\
\text { são os fatores mais } \\
\text { determinantes para } \\
\text { a QV. }\end{array}$ \\
\hline 7 & $\begin{array}{l}2016 \\
\text { EUA }\end{array}$ & $\begin{array}{l}\text { Quality of life } \\
\text { in united states } \\
\text { veterans with } \\
\text { combat-related } \\
\text { ostomies from Iraq } \\
\text { and Afghanistan }\end{array}$ & $\begin{array}{l}\text { Sto- } \\
\text { ma-QoL } \\
\text { and } \\
\text { Veterans } \\
\text { RAND 36 } \\
\text { scores }\end{array}$ & Trauma & $\begin{array}{c}\text { A estomia tem um } \\
\text { impacto mínimo de } \\
\text { longo prazo na QV do } \\
\text { paciente em vítimas de } \\
\text { combate. }\end{array}$ \\
\hline 8 & $\begin{array}{l}2016 \\
\text { Brasil }\end{array}$ & $\begin{array}{l}\text { Convivendo com } \\
\text { uma estomia } \\
\text { úmida: um estudo } \\
\text { sobre qualidade } \\
\text { de vida }\end{array}$ & $\begin{array}{l}\text { WHO } \\
\text { QOL- } \\
\text { BREF }\end{array}$ & $\begin{array}{l}\text { Neoplasia } \\
\text { retal }\end{array}$ & $\begin{array}{l}\text { Uma melhor QV pode } \\
\text { ser adquirida com } \\
\text { atividades que tragam } \\
\text { prazer e motivação. }\end{array}$ \\
\hline 9 & $\begin{array}{l}2017 \\
\text { Brasil }\end{array}$ & $\begin{array}{l}\text { Oncology ostomized } \\
\text { patients' perception } \\
\text { regarding sexual } \\
\text { relationship as } \\
\text { an important } \\
\text { dimension in quality } \\
\text { of life }\end{array}$ & $\begin{array}{l}\text { WHO } \\
\text { QOL- } \\
\text { BREF }\end{array}$ & $\begin{array}{l}\text { Neoplasia de } \\
\text { intestino }\end{array}$ & $\begin{array}{c}\text { A sexualidade deve } \\
\text { ser considerada um } \\
\text { processo de vida diário } \\
\text { para o paciente, res- } \\
\text { saltando a importância } \\
\text { de sua correlação com } \\
\text { a prática profissional, } \\
\text { resultando em melhoria } \\
\text { da QV. }\end{array}$ \\
\hline 10 & $\begin{array}{l}2017 \\
\text { Brasil }\end{array}$ & $\begin{array}{l}\text { Self-esteem and } \\
\text { health-related } \\
\text { quality of life in } \\
\text { ostomized patients }\end{array}$ & $\begin{array}{c}\text { EAR; } \\
\text { EORTC } \\
\text { QLQ-C30 }\end{array}$ & $\begin{array}{l}\text { Neoplasia } \\
\text { colorretal }\end{array}$ & $\begin{array}{l}\text { A autoestima foi con- } \\
\text { siderada satisfatória. }\end{array}$ \\
\hline 11 & $\begin{array}{l}2017 \\
\text { Brasil }\end{array}$ & $\begin{array}{l}\text { Qualidade de } \\
\text { vida de pessoas } \\
\text { com estomias } \\
\text { intestinais de } \\
\text { eliminação }\end{array}$ & $\begin{array}{c}\mathrm{COH} \\
\mathrm{QOL-OQ}\end{array}$ & Não referido & $\begin{array}{l}\text { As estomias intestinais } \\
\text { interferem na QV, prin- } \\
\text { cipalmente nos âmbitos } \\
\text { físico e social. }\end{array}$ \\
\hline 12 & $\begin{array}{l}2017 \\
\text { Brasil }\end{array}$ & $\begin{array}{l}\text { Perceptions of } \\
\text { ostomized persons } \\
\text { due to colorectal } \\
\text { cancer on their } \\
\text { quality of life }\end{array}$ & $\begin{array}{l}\text { WHO } \\
\text { QOL- } \\
\text { BREF }\end{array}$ & $\begin{array}{l}\text { Neoplasia } \\
\text { colorretal }\end{array}$ & $\begin{array}{l}\text { O uso de um equipa- } \\
\text { mento coletor pode } \\
\text { representar a mutilação } \\
\text { sofrida, comprometen- } \\
\text { do a QV. }\end{array}$ \\
\hline
\end{tabular}

\begin{tabular}{|c|c|c|c|c|c|}
\hline 13 & $\begin{array}{l}2017 \\
\text { Holanda }\end{array}$ & $\begin{array}{l}\text { Quality of life in } \\
\text { elderly patients } \\
\text { with an ostomy - a } \\
\text { study from the } \\
\text { population-based } \\
\text { PROFILES registry }\end{array}$ & $\begin{array}{l}\text { EORTC } \\
\text { QLQ-C30 }\end{array}$ & $\begin{array}{l}\text { Neoplasia } \\
\text { colorretal }\end{array}$ & $\begin{array}{c}\text { O impacto na QV } \\
\text { causado pela estomia } \\
\text { parece ser mais pro- } \\
\text { eminente nos pacientes } \\
\text { mais jovens. }\end{array}$ \\
\hline 14 & $\begin{array}{l}2017 \\
\text { China }\end{array}$ & $\begin{array}{l}\text { Determinants of } \\
\text { self-efficacy and } \\
\text { quality of life in } \\
\text { patients with tem- } \\
\text { porary enterostomy: } \\
\text { a cross-sectional } \\
\text { survey }\end{array}$ & $\begin{array}{l}\text { Stoma } \\
\text { Self-Effi- } \\
\text { cacy Scale } \\
\text { and COH } \\
\text { QOL-OQ }\end{array}$ & $\begin{array}{l}\text { Neoplasia } \\
\text { colorretal }\end{array}$ & $\begin{array}{l}\text { A QV global foi de- } \\
\text { terminada por tipo de } \\
\text { enterostomia e método } \\
\text { de pagamento do trata- } \\
\text { mento de saúde. }\end{array}$ \\
\hline 15 & $\begin{array}{l}2018 \\
\text { Brasil }\end{array}$ & $\begin{array}{l}\text { Quality of life of } \\
\text { ostomized person: } \\
\text { relationship with } \\
\text { the care provided in } \\
\text { stomatherapy nurs- } \\
\text { ing consultation }\end{array}$ & $\begin{array}{c}\mathrm{COH} \\
\mathrm{QOL-OQ}\end{array}$ & $\begin{array}{l}\text { Neoplasia, } \\
\text { fistula, } \\
\text { perfuração } \\
\text { intestinal, } \\
\text { diverticulite, } \\
\text { doença } \\
\text { inflamatória } \\
\text { intestinal }\end{array}$ & $\begin{array}{c}\text { Existe relação } \\
\text { estatistica entre QV, } \\
\text { o tipo de estomia e } \\
\text { participação na consul- } \\
\text { ta de enfermagem de } \\
\text { estomaterapia, demon- } \\
\text { strando a influência } \\
\text { positiva dos cuidados } \\
\text { de Enfermagem. }\end{array}$ \\
\hline 16 & $\begin{array}{l}2018 \\
\text { Sérvia }\end{array}$ & $\begin{array}{l}\text { Psychological and } \\
\text { spiritual well-being } \\
\text { aspects of the } \\
\text { quality of life in } \\
\text { colostomy patients }\end{array}$ & $\begin{array}{c}\mathrm{COH} \\
\mathrm{QOL-OQ}\end{array}$ & $\begin{array}{l}\text { Neoplasia } \\
\text { colorretal }\end{array}$ & $\begin{array}{l}\text { É necessário prevenção } \\
\text { e tratamento de } \\
\text { reações negativas em } \\
\text { relação ao estoma e } \\
\text { melhoria da QV. }\end{array}$ \\
\hline 17 & $\begin{array}{l}2018 \\
\text { Turquia }\end{array}$ & $\begin{array}{l}\text { The effect of } \\
\text { preoperative stoma } \\
\text { site marking on } \\
\text { quality of life }\end{array}$ & $\begin{array}{c}\mathrm{COH} \\
\mathrm{QOL-OQ}\end{array}$ & $\begin{array}{l}\text { Neoplasia } \\
\text { colorretal }\end{array}$ & $\begin{array}{l}\text { A marcação do local } \\
\text { do estoma aumenta } \\
\text { a QVRS durante o } \\
\text { pós-operatório. }\end{array}$ \\
\hline
\end{tabular}

Quadro 4. Níveis de Evidência dos Estudos Dados da pesquisa, 2019.

\begin{tabular}{|c|c|c|c|c|}
\hline $\mathrm{N}^{\circ}$ & Ano & Título & $\begin{array}{l}\text { Tipo de } \\
\text { Estudo }\end{array}$ & $\begin{array}{l}\text { Nível de } \\
\text { Evidência }\end{array}$ \\
\hline I & 2014 & $\begin{array}{l}\text { Quality of life and self-esteem of } \\
\text { patients with intestinal stoma }\end{array}$ & Descritivo & VI \\
\hline 2 & 2014 & $\begin{array}{l}\text { The influence of time on the } \\
\text { quality of life of patients with } \\
\text { intestinal stoma }\end{array}$ & Descritivo & VI \\
\hline 3 & 2015 & $\begin{array}{l}\text { A mixed-method study on the } \\
\text { generic and ostomy-specific } \\
\text { quality of life of cancer and } \\
\text { non-cancer ostomy patients }\end{array}$ & Descritivo & VI \\
\hline 4 & 2016 & $\begin{array}{l}\text { Quality of life in stomized onco- } \\
\text { logical patients: an approach of } \\
\text { integrality from Brazilian Unified } \\
\text { Health System }\end{array}$ & Descritivo & VI \\
\hline 5 & 2016 & $\begin{array}{l}\text { Sociodemographic and clinical } \\
\text { features and quality of life in } \\
\text { stomized patients }\end{array}$ & Descritivo & $\mathrm{VI}$ \\
\hline 6 & 2016 & $\begin{array}{l}\text { Quality of life and need for care } \\
\text { in patients with an ostomy: a sur- } \\
\text { vey of } 2647 \text { patients of the Berlin } \\
\text { OStomy-Study (BOSS) }\end{array}$ & Descritivo & VI \\
\hline 7 & 2016 & $\begin{array}{l}\text { Quality of life in united states } \\
\text { veterans with combat-related os- } \\
\text { tomies from Iraq and Afghanistan }\end{array}$ & Descritivo & VI \\
\hline 8 & 2016 & $\begin{array}{l}\text { Convivendo com uma estomia } \\
\text { úmida: um estudo sobre quali- } \\
\text { dade de vida }\end{array}$ & Descritivo & VI \\
\hline 9 & 2017 & $\begin{array}{l}\text { Oncology ostomized patients' } \\
\text { perception regarding sexual } \\
\text { relationship as an important } \\
\text { dimension in quality of life }\end{array}$ & Descritivo & $\mathrm{VI}$ \\
\hline 10 & 2017 & $\begin{array}{l}\text { Self-esteem and health-related } \\
\text { quality of life in ostomized } \\
\text { patients }\end{array}$ & Descritivo & VI \\
\hline II & 2017 & $\begin{array}{l}\text { Qualidade de vida de pessoas } \\
\text { com estomias intestinais de } \\
\text { eliminação }\end{array}$ & Descritivo & VI \\
\hline 12 & 2017 & $\begin{array}{l}\text { Perceptions of ostomized per- } \\
\text { sons due to colorectal cancer on } \\
\text { their quality of life }\end{array}$ & Descritivo & VI \\
\hline 13 & 2017 & $\begin{array}{l}\text { Quality of life in elderly patients } \\
\text { with an ostomy - a study from } \\
\text { the population-based PROFILES } \\
\text { registry }\end{array}$ & Descritivo & VI \\
\hline
\end{tabular}




\begin{tabular}{|l|l|l|l|l|}
\hline 14 & 2017 & $\begin{array}{l}\text { Determinants of self-efficacy } \\
\text { and quality of life in patients } \\
\text { with temporary enterostomy: a } \\
\text { cross-sectional survey }\end{array}$ & Descritivo & $\mathrm{VI}$ \\
\hline 15 & 2018 & $\begin{array}{l}\text { Quality of life of ostomized } \\
\text { person: relationship with the care } \\
\text { provided in stomatherapy nursing } \\
\text { consultation }\end{array}$ & Descritivo & $\mathrm{VI}$ \\
\hline 16 & 2018 & $\begin{array}{l}\text { Psychological and spiritual } \\
\text { well-being aspects of the quality } \\
\text { of life in colostomy patients }\end{array}$ & Descritivo & $\mathrm{VI}$ \\
\hline 17 & 2018 & $\begin{array}{l}\text { The effect of preoperative stoma } \\
\text { site marking on quality of life }\end{array}$ & $\begin{array}{l}\text { Ensaios } \\
\text { clínicos bem } \\
\text { desenhados } \\
\text { sem rand- } \\
\text { omização }\end{array}$ & $\mathrm{III}$ \\
\hline
\end{tabular}

\section{DISCUSSÃO}

Nesse estudo foram identificados sete instrumentos utilizados para avaliar a QV das pessoas com estomias intestinais. Alguns instrumentos encontrados são específicos para esse público, sendo eles: COHQOL-OQ, Stoma-QoL e SSES, enquanto os outros são gerais: WHOQOL-Bref,EORTC QLQ-C30, Veterans RAND-36 e Escala de Qualidade de Vida de Flanagan.

Esses instrumentos de avaliação são importantes, pois, a partir dos resultados obtidos, pode-se adotar medidas de intervenção que sejam eficazes, a fim de melhorar a QV das pessoas com estomias intestinais. Cada um deles possui suas particularidades, que serão discutidas abaixo.

\section{Escala de Qualidade de Vida de Flanagan (EQVF)}

A EQVF foi criada na década de 70, por John Flanagan e já está traduzida para diversos idiomas ${ }^{(16)}$. $O$ instrumento possui respostas em escala de Likert de I a 7 e avalia a QV de forma multidimensional, a partir de 15 itens, representando cinco domínios conceituais: bem-estar físico e material, relações com outras pessoas, atividades sociais e cívicas, desenvolvimento pessoal e recreação(16,17).

O item bem-estar refere-se à boa disposição física, conforto e tranquilidade material; o de relacionamento compreende a ligação afetiva, condicionada por várias atitudes recíprocas; - de atividade envolve comportamentos emitidos como resposta ao desenvolver atividades de lazer, como clubes da terceira idade, ou ao exercer ações de caráter mais político, como as associações de aposentados; o desenvolvimento pessoal consiste na autoaceitação, relações positivas com outros, autonomia, senso de domínio e busca de metas; e o de recreação compreende as atividades de distração individual e social(17).

A EQVF é mais utilizada em idosos, pois apresenta e esclarece vários aspectos da vida desse público, em especial os de caráter intersubjetivo, com maior possibilidade de ocorrência na terceira idade, como acometimento de doenças em geral, perda de papéis ocupacionais e perdas afetivas ${ }^{(18)}$. Esse fato justifica que a EQVF tenha sido encontrada em apenas um estudo.

No entanto, ressalta-se que as pessoas com estomias intestinais vivenciam situações semelhantes, já que as modificações consequentes à estomia envolvem alterações emocionais, dificuldade de desenvolver novos relacionamentos, atitudes de isolamento e vergonha de expor-se ${ }^{(19)}$. Essas situações, dentre muitas outras, também levam às perdas de papéis sociais e afetivos, alterando a QV das pessoas com estomias intestinais.
World Health Organization Quality of Life abreviado (WHOQOLBref)

O WHOQOL-Bref é uma versão abreviada do World Health Organization Quality of Life (WHOQOL-I00). O WHOQOL-100 foi apresentado pela Organização Mundial da Saúde em 1998, e busca avaliar a QV em diferentes cenários culturais, além de possibilitar a comparação direta dos resultados obtido nestas diferentes configurações. $O$ instrumento consiste em cem perguntas referentes a seis domínios: físico, psicológico, nível de independência, relações sociais, meio ambiente e espiritualidade ${ }^{(20)}$.

Esses domínios são divididos em 24 facetas, com quatro perguntas cada, além de uma 25 a faceta com perguntas gerais sobre QV. As respostas para as questões do WHOQOL-100 são dadas em uma escala do tipo Likert. As perguntas são respondidas através de quatro tipos de escalas: intensidade, capacidade, frequência e avaliação, que variam de acordo com o conteúdo da pergunta ${ }^{(20)}$.

A partir disso, foi desenvolvido o WHOQOL-Bref, pela necessidade de um instrumento que mantivesse as características psicométricas satisfatórias do WHOQOL-100, mas demandasse menos tempo para o preenchimento. $O$ WHOQOL-Bref contém 26 questões, sendo duas questões gerais de $\mathrm{QV}$, e 24 facetas que compõem o instrumento original(20).

No entanto, o WHOQOL-Bref possui apenas uma pergunta em cada faceta, representando o conceito de origem de cada uma delas, e não quatro, como no instrumento original. Assim, - WHOQOL-bref é composto pelos domínios: físico, psicológico, relações sociais e meio ambiente ${ }^{(20)}$.

O WHOQOL-Bref, como um instrumento genérico, é aplicados na avaliação da QV da população em geral, sem especificação de enfermidades, sendo mais apropriado para estudos epidemiológicos, planejamento e avaliação do sistema de saúde. O WHOQOL-I00 é um dos instrumentos genéricos de avaliação de $\mathrm{QV}$ mais frequentemente utilizados no mundo. No entanto, como WHOQOL-bref não compromete a qualidade dos resultados quando comparado ao WHOQOL-100. Assim, - WHOQOL-bref vem sendo amplamente utilizado, a fim de tornar a aplicação e avaliação mais prática e com características psicométricas satisfatórias ${ }^{(21)}$.

\section{Ostomy-specific (Stoma-QoL)}

O Stoma-QoL foi criado em 2005, a partir da necessidade de se avaliar a QV com a especificidade das pessoas com estomias, resultando de um questionário com 37 questões, aplicado por enfermeiras estomaterapeutas em 182 pacientes de quatro diferentes países europeus e com causas variadas para a criação de um estoma. O conteúdo original do questionário, que derivou no instrumento, é proveniente de entrevistas qualitativas com pacientes com estoma, para determinar as reais necessidades dos pacientes, e não partir de pesquisas na literatura ou em análises de especialistas, como comumente são feitos os instrumentos ${ }^{(22)}$.

Após a aplicação para validação, o Stoma-QoL resultou em um questionário de 20 itens, dentro dos quatro domínios: sono, atividade sexual, relações com familiares e amigos íntimos e relações sociais com outros que não a família e amigos íntimos. As respostas são dadas de acordo com o nível de concordância, em uma escala Likert de quatro pontos ${ }^{(22)}$.

Uma pontuação total é calculada a partir da soma de todas as pontuações dos itens, sendo convertida em uma pontuação de 0 a 100, em que uma pontuação alta representa um nível 
mais alto de QV e um nível mais baixo de sintomas, enquanto pontuações mais baixas representam baixos níveis de QV e níveis altos de sintomas ${ }^{(22)}$

Por ser um instrumento específico, o Stoma-QoL tem forte evidência, em comparação com outros instrumentos genéricos, já que sua sensibilidade é aumentada para os problemas específicos de uma condição específica, sendo nesse caso, a estomização. No entanto, instrumentos específicos também possuem limitações e o Stoma-QoL não é abrangente fora de seus quatro domínios finais, não podendo ser utilizado para comparar com outras condições além do estoma ${ }^{(22)}$.Além disso, esse instrumento não abrange pessoas com estomia urinária, sendo utilizado apenas em pessoas com estomias intestinais (ileostomias e colostomias).

City of Hope Quality of Life-Ostomy Questionnaire (COHQOL-OQ)

Desenvolvido por Grant e colaboradores em 2004, a partir de outro instrumento da década de 80 , dos próprios autores, intitulado City of Hope - Quality of Life - Colostomy pacientes. A expansão do instrumento visava incluir pacientes com estomias intestinais e urinárias, com e sem câncer e não apenas colostomias ${ }^{(23)}$.

O COH-QOL-OQ é uma avaliação específica para pessoas com estomias, com 90 questões divididas em 3 seções: características demográficas e clínicas, impacto de estomas no estilo de vida e impacto na QV. A última seção consiste em 43 itens e conceitua QV usando quatro domínios: bem-estar físico, bem psicológico, bem-estar social e bem-estar espiritual. Os escores de domínio variam de 0 a 10 , e escores mais altos indicam melhor $\mathrm{QV}^{(23,24)}$.

No Brasil, a versão em língua portuguesa do COHQOL-OQ é o primeiro instrumento específico para medir a $\mathrm{QV}$ relacionada à saúde (QVRS) em pessoas que vivem com uma estomia(13). É importante ressaltar que o $\mathrm{COH}-\mathrm{QOL}-\mathrm{OQ}$ é o instrumento mais abrangente, devido ao elevado número de itens avaliados - 43 itens -, em comparação aos outros instrumentos encontrados nos estudos analisados. Além disso, é um instrumento capaz de avaliar a QV de pessoas com estomias urinárias ou intestinais, com ou sem neoplasia. Essas características justificam o fato desse instrumento específico ser o mais utilizado dentre os estudos encontrados.

\section{Stoma Self-Efficacy Scale (SSES)}

A SSES é utilizada para examinar a auto-eficácia de pessoas com estomia. A autoeficácia relaciona-se ao discernimento pessoal dos indivíduos acerca de sua própria capacidade de organização e implementação de atividades, em situações desconhecidas, com elementos ambíguos, imprevisíveis ou geradores de estresse.A percepção de eficácia pode ter efeitos diversos no comportamento, nos padrões de pensamento, e nos aspectos emocionais ${ }^{(25)}$.

Esse instrumento é composto por 22 itens, subdivididos em autoeficácia do tratamento estomacal, que mede a capacidade pessoal de cuidar de seu estoma, com 13 itens e autoeficácia social, que mede a autoeficácia da pessoa em relação ao seu estoma, com 9 itens. $O$ instrumento é respondido por meio de escala Likert de 5 pontos, onde um é não confiante e cinco extremamente confiante. A pontuação mínima é 22 e a máxima 110 . Escores mais altos obtidos nas pontuações indicam melhor autoeficácia em relação ao estoma(26).

\section{Veterans RAND-36}

O Inquérito de Saúde Veteran RAND 36 (VR-36) foi desenvolvido a partir do Modelo MOS RAND-36 Versão I.0 (RAND Corporation, Santa Monica, Califórnia) ${ }^{(27)}$. Este instrumento possui 36 questões relacionadas à saúde e já foi relatado como sendo um instrumento válido e confiável para ser utilizado em uma população de veteranos de guerra ${ }^{(28)}$. Os escores totais por construto foram convertidos para um escore padrão de 0 a 100 , em que um número maior indicou uma melhor QV.

European Organization for Research and Treatment of Cancer Quality of Life Questionnaire (EORTC QLQ-C30)

A EORTC é um dos maiores e mais antigos grupos de estudos clínicos da Europa e desenvolveu um questionário sobre qualidade de vida específico para o câncer (QLQ). O primeiro surgiu em 1987, e a versão mais recente do questionário, conhecido como EORTC QLQ-C30 versão 2.0, tem sido disseminada pela European Organization for Research and Treatment of Cancer desde a sua publicação(29,30).

O EORTC QLQ-C30 é um questionário de 30 itens específico para medição da QV em pacientes com câncer. Compreende cinco escalas funcionais (físico, papel, cognitivo, emocional e social), três escalas de sintomas (fadiga, dor e náuseas e vômitos), e uma escala de saúde e QV(31).

Os itens únicos restantes avaliam sintomas adicionais comumente relatados por pacientes com câncer (dispneia, perda de apetite, perturbação do sono, obstipação e diarreia) e também o impacto financeiro percebido da doença e tratamento. $O$ intrumento é respondido por meio de escala Likert de 4 pontos ${ }^{(31)}$.

O EORTC QLQ-C30, ainda que forneça uma riqueza de informações sobre a QVRS dos pacientes, também se apresenta como um desafio analítico, devido aos múltiplos resultados que procedem da aplicação desse instrumento. Sendo assim, portanto, essa é uma limitação significativa do EORTC QLQ-C30.

Alguns estudos o utilizam definindo um conjunto limitado de escalas funcionais, que sejam de interesse primário. No entanto, pode ser difícil pré-definir quais escalas do EORTC QLQ-C30 são de maior interesse. Em tais casos, os pesquisadores comumente utilizam a escala de dois itens, que avalia a QV geral(32). No entanto, nesses casos, não há especificidade para o público estudado, além de apresentar como desvantagem a imprecisão da medição de $\mathrm{QV}$ e pode não ser um resumo adequado do instrumento, que possui um número relativamente grande de escalas e itens de sintomas.

Por fim, é importante ressaltar que o Brasil se destaca na produção científica acerca da temática, podendo ser evidenciado pelo número de artigos encontrados na presente revisão. Da mesma forma, um estudo sobre as evidências científicas relativas às pessoas com estomias intestinais definitivas decorrentes de câncer colorretal e as repercussões para a QV dessas pessoas, concluiu que sete $(30,4 \%)$ dos 23 estudos encontrados foram realizados no Brasil( ${ }^{(33)}$.

\section{CONCLUSÃO}

Esta revisão permitiu identificar os instrumentos que estão sendo utilizados para avaliar a QV de pessoas com estomias intestinais. Essa avaliação é de suma importância para as pessoas com estomias intestinais, já que assim podese nortear as intervenções adequadas a esse público, a fim de auxiliar na melhora da QV. Com base nas características dos 
instrumentos, nota-se que é possível avaliar a QV de pessoas intestinais com instrumentos genéricos.

No entanto, os instrumentos específicos abordam questões particulares das pessoas com estomias intestinais, sendo esses os mais utilizados pelos estudos abordados nesta revisão.Após a análise atenta de todos os instrumentos anteriormente referidos, salienta-se que o City of Hope - Quality of Life - Ostomy Questionnaire é o mais abrangente dentre os instrumentos específicos às pessoas com estomias, sendo o único a incluir o domínio espiritual na avaliação de QV e é também o mais utilizado nos estudos encontrados nesta revisão.

Acredita-se que seja necessário a realização de novos estudos de avaliação de QV de pessoas com estomias intestinais para que se tenha maior subsídio para a elaboração de intervenções voltadas à melhora da QV dessas pessoas.

\section{REFERÊNCIAS}

1. Mcclees N, Hyland D, Bolton L. Quality Of Life With An Ostomy: Historic Perspective 1444. J Wound Ostomy Cont Nurs. 2007; 34(72). Disponível em: https:// journals.Iww.com/jwocnonline/Citation/2007/0500 I/ QUALITY_OF_LIFE_WITH_AN_OSTOMY HISTORIC.203.aspx

2. Auquier P, Simeoni MC, Mendizabal H. Approches théoriques et méthodologiques de la qualité de vie liée à la santé. Revue Prevenir.1997; 33: 77-86.

3. Fleck MPA, et al. Desenvolvimento da versão em português do instrumento de avaliação de Qualidade de Vida da OMS (WHOQOL-100). Revista Brasileira de Psiquiatria. 1999; 2I(I): 19- 28. Disponível em: http://www.scielo.br/pdf/rbp/v2 I n I/v2 I n la06.pdf

4. The Whogol Group. The development of the World Health Organization quality of life assessment instrument (the WHOQOL). In: Orley J, Kuyken W, editors. Quality of life assessment: international perspectives. Heidelberg: Springer-Verlag. 1994: 4l-60. Disponível em: https://link.springer.com/ chapter/10.1007/978-3-642-79/23-9_4

5. Bullinger M, Anderson R, Cella D. Developing anda evaluating cross-cultural instruments from minimum requirements to optimal models. Qual Life Res.1993; 2(6): 45I-9. Disponível em: https://link.springer.com/ article/I0.1007/BF00422219

6. Fleck MPA. O instrumento de avaliação de qualidade de vida da Organização Mundial de Saúde (WHOQOL-100): características e perspectivas. Revista Ciência e Saúde Coletiva. 2000; 5(I): 3338. Disponível em: http://www.scielo.br/pdf/csc/ v5n I/7077.pdf

7. Brasil. Ministério da Saúde. Portaria SAS/MS n 400 de 16 de novembro de 2009 [Internet]. Brasília, 2009 [Citado dez 2014]. Disponível em: http://bvsms.saude. gov.br/bvs/saudelegis/sas/2009/prt0400_I6_II_2009. html.

8. ROCHA, J.J.R. Estomas intestinais (ileostomias e colostomias) e anastomoses intestinais Med (Ribeirão Preto). 20II.

9. Mota MS, Gomes GC, Silva CD, Gomes VL, Pelzer MT, Barros E.Autocuidado: uma estratégia para a qualidade de vida da pessoa com estomia. Investig Enferm. Imagen Desarr. 2016; I8(I):63-78. Disponível em: http://dx.doi. org/ I0.I I |44/Javeriana.ie I8- I.aeqv
10. Mota MS, Gomes GC, PetucoVM, Heck RM, Barros EJL, \& De Oliveira Gomes VL. Facilitadores do processo de transição para o autocuidado da pessoa com estoma: subsídios para Enfermagem. Revista da Escola de Enfermagem da USP, v. 49, n. I, p. 82-88, 2015. Disponível em: https://www.revistas.usp.br/reeusp/ article/view/I03164

11. Keszei AP, Novak M, Streiner DL. Introduction to health measurement scales. Journal of Psychosomatic Research. 2010; 68(4): 319-23. Disponível em: https:// www.sciencedirect.com/science/article/abs/pii/ S00223999I0000II5

12. Reppold CT, Gurgel LG, Hutz CS. O processo de construção de escalas psicométricas. Avaliação Psicológica. 20 I4; I3(2):.307-3 I0. Disponível em: http:// pepsic.bvsalud.org/pdf/avp/v13n2/v|3n2al8.pdf

13. Santos VLCG, Augusto F, Gomboski G. HealthRelated Quality of Life in Persons With Ostomies Managed in an Outpatient Care Setting. Journal of Wound, Ostomy and Continence Nursing. 2016;43(2): 158-164. Disponível em: https://insights.ovid.com/ pubmed?pmid=26808303

14. Padilla GV, Grant MM. Quality of life as a cancer nursing outcome variable. ANS Adv Nurs Sci. 1985; 8(I): 45-60. Disponível em: https://psycnet.apa.org/ record/|986-|3452-00 |

15. Mendes KDS, Silveira RCDCP, Galvão CM. Revisão integrativa: método de pesquisa para a incorporação de evidências na saúde e na enfermagem. Texto \& contexto enfermagem. 2008; I7(4): 758-64. Disponível em: http://www.scielo.br/pdf/tce/vI7n4//8.pdf

16. Burckhardt CS, Anderson KL. The Quality of Life Scale (QOLS): Reliability, Validity, and Utilization. Health and Quality of Life Outcomes.2003; I(I): I-7. Disponível em: https://www.ncbi.nlm.nih.gov/pmc/ articles/PMC269997/pdf/I477-7525-I-60.pdf

17. Aerosa SVC, Benitez LB, Wichmann FMA. Relações familiares e o convívio social entre idosos. Textos Contextos.2012; II(I): I84-92. Disponível em: http:// revistaseletronicas.pucrs.br/ojs/index.php/fass/article/ viewFile/ I0495/8059

18. Oliveira BC, et al. Avaliação da qualidade de vida em idosos da comunidade. Revista Brasileira em Promoção da Saúde. 2017; 30(3): I-I0. Disponível em: https:// periodicos.unifor.br/RBPS/article/view/5879/pdf

19. Cardoso DBR, et al. Sexualidade de pessoas com estomias intestinais. Revista da Rede de Enfermagem do Nordeste.2015; 16(4):. 576-85. Disponível em: http://periodicos.ufc.br/rene/article/view/2750/2I33

20. The Whoqol Group. The World Health Organization quality of life assessment (WHOQOL): Development and general psychometric properties. Social Science \& Medicine. 1998; 46(12): 1569-85. Doi:I0.1016/s02779536(98)00009-4

21. Ferreira JC, Romero VU, De Andrade RM, Sá EC. Avaliação da qualidade de vida de açougueiros com a utilização do WHOQOL-bref. Rev. bras. med. trab. 2017; 15(3): 222-28. Disponível em: http://www.rbmt. org.br/details/252/pt-BR/avaliacao-da-qualidade-devida-de-acougueiros-com-a-utilizacao-do-whoqol-bref

22. Prieto L.Thorsen H, Juul K. Development and validation of quality of life patients with colostomy or ileostomy. 
Health and quality of life outcomes. 2005;.3(62): I-I0. Disponível em: https://www.ncbi.nlm.nih.gov/pmc/ articles/PMC I 274339/pdf/ |477-7525-3-62.pdf

23. Grant $M$, et al. Revision and psychometric testing of the City of Hope Quality of Life-Ostomy Questionnaire. Quality of Life Research. 2004; 13(8): |445-|457. Disponível em: https://link.springer.com/ article/ I0. I023/B:QURE.0000040784.65830.9f

24. Gomboski G. Adaptação cultural e validação do City of Hope-Quality of Life-Ostomy Questionaire para a língua portuguesa no Brasil. Tese de Doutorado.São Paulo: Universidade de São Paulo; 2010. Disponível em: http://www.teses.usp.br/teses/disponiveis/7/7139/tde010820 I I-074203/pt-br.php

25. Ribeiro JLP. Adaptação de uma Escala de Avaliação da Auto-eficácia Geral. Faculdade de Psicologia e de Ciências da Educação. Porto; 2004. https://www.fpce. up.pt/docentes/paisribeiro/testes/EFICACIA.htm

26. Bekkers MJTM, Van Knippenber FCEV, Van Den Borne HW, Van Berge-Henegouwen GP. Prospective evaluation of psychosocial adaptation to stoma surgery: the role of self-efficacy. Psychosom Med. 1996; 58(2): |83-91, 1996. Disponível em: https://insights.ovid.com/ pubmed?pmid $=8849636$

27. Hays RD, Sherbourne CD, Mazel RM.The rand 36-item health survey 1.0. Health economics. 1993; 2(3): 217227. Disponível em: https://onlinelibrary.wiley.com/ doi/abs/ I 0.1002/hec.4730020305

28. Kazis LE, et al. Patient-reported measures of health: the Veterans Health Study. J Ambul Care Manage. 2004; 27(I): 70-83. Disponível em: https://journals.Iww.com/ ambulatorycaremanagement/Abstract/2004/01000/ Patient_Reported_Measures_of_Health__The_ Veterans. I2.aspx
29. Aaronson NK, et al. The eortc quality of life questionnaire: interim results of an international field study. In: Osoba D (ed) Effects of cancer on quality of life. CRC Press, Boca Raton. 1991; 185-203. Disponível em: https://difusion.ulb.ac.be/vufind/Record/ULBDIPOT:oai:dipot.ulb.ac.be:2013/66657/Details

30. Fayers P,Aaronson N,Bjordal , Sullivan M. EORTC-C30 scoring manual. EORTC Study Group on Quality of Life, Brussels, 1995.

31. Aaronson NK, Ahmedzai S, Bergman B, et al. The European Organization for Research and Treatment of Cancer QLQ-C30: a quality of life instrument for use in international clinical trials in oncology.J Natl Cancer Inst. 1993; 85(5): 365-376. Disponível em: https://academic. oup.com/jnci/article-abstract/85/5/365/972260

32. Giesinger JM, et al. Replication and validation of higher order models demonstrated that a summary score for the EORTC QLQ-C30 is robust. Journal of clinical epidemiology. 2016; 69: 79-88. Disponível em: https:// www.jclinepi.com/article/S0895-4356(I 5)00383-2/pdf

33. Maciel DBV, Dos Santos MLSC, Souza NVDO, Fuly PDSC, Camacho ACLF, Soares HPL. Qualidade de Vida de Pessoas com Estomias Intestinais Definitivos: uma Revisão Integrativa. Revista Enfermagem Atual InDerme. 2018; 2018(86). Disponível em: http:// revistaenfermagematual.com/index.php/revista/article/ view/109
Recebido: 2019-08-16 Aceito: 2019-10-04 